\title{
Resection of tumors within the primary motor cortex using high-frequency stimulation: oncological and functional efficiency of this versatile approach based on clinical conditions
}

\author{
*Marco Rossi, MD,1 Marco Conti Nibali, MD,'1 Luca Viganò,, Guglielmo Puglisi, PhD, ${ }^{2}$ \\ Henrietta Howells, PhD, ${ }^{2}$ Lorenzo Gay, MD, ${ }^{1}$ Tommaso Sciortino, MD, ${ }^{1}$ Antonella Leonetti, PhD, ${ }^{2}$ \\ Marco Riva, MD, ${ }^{3}$ Luca Fornia, PhD, ${ }^{2}$ Gabriella Cerri, PhD, ${ }^{2}$ and Lorenzo Bello, MD1 \\ ${ }^{1}$ Neurosurgical Oncology Unit, Department of Oncology and Hemato-Oncology; ${ }^{2}$ Laboratory of Motor Control, Department of \\ Medical Biotechnology and Translational Medicine; and ${ }^{3}$ Department of Medical Biotechnology and Translational Medicine, \\ Università degli Studi di Milano and Humanitas Research Hospital, IRCCS, Milano, Italy
}

\begin{abstract}
OBJECTIVE Brain mapping techniques allow one to effectively approach tumors involving the primary motor cortex (M1). Tumor resectability and maintenance of patient integrity depend on the ability to successfully identify motor tracts during resection by choosing the most appropriate neurophysiological paradigm for motor mapping. Mapping with a highfrequency (HF) stimulation technique has emerged as the most efficient tool to identify motor tracts because of its versatility in different clinical settings. At present, few data are available on the use of HF for removal of tumors predominantly involving M1.
\end{abstract}

METHODS The authors retrospectively analyzed a series of 102 patients with brain tumors within M1, by reviewing the use of HF as a guide. The neurophysiological protocols adopted during resections were described and correlated with patients' clinical and tumor imaging features. Feasibility of mapping, extent of resection, and motor function assessment were used to evaluate the oncological and functional outcome to be correlated with the selected neurophysiological parameters used for guiding resection. The study aimed to define the most efficient protocol to guide resection for each clinical condition.

RESULTS The data confirmed HF as an efficient tool for guiding resection of M1 tumors, affording $85.3 \%$ complete resection and only $2 \%$ permanent morbidity. HF was highly versatile, adapting the stimulation paradigm and the probe to the clinical context. Three approaches were used. The first was a "standard approach" (HF "train of 5," using a monopolar probe) applied in 51 patients with no motor deficit and seizure control, harboring a well-defined tumor, showing contrast enhancement in most cases, and reaching the M1 surface. Complete resection was achieved in $72.5 \%$, and $2 \%$ had permanent morbidity. The second approach was an "increased train approach," that is, an increase in the number of pulses (7-9) and of pulse duration, using a monopolar probe. This second approach was applied in 8 patients with a long clinical history, previous treatment (surgery, radiation therapy, chemotherapy), motor deficit at admission, poor seizure control, and mostly high-grade gliomas or metastases. Complete resection was achieved in $87.5 \%$ using this approach, along with $0 \%$ permanent morbidity. The final approach was a "reduced train approach," which was the combined use of train of 2 or train of 1 pulses associated with the standard approach, using a monopolar or bipolar probe. This approach was used in 43 patients with a long clinical history and poorly controlled seizures, harboring tumors with irregular borders without contrast enhancement (low or lower grade), possibly not reaching the cortical surface. Complete resection was attained in $88.4 \%$, and permanent morbidity was found in $2.3 \%$.

CONCLUSIONS Resection of M1 tumors is feasible and safe. By adapting the stimulation paradigm and probe appropriately to the clinical context, the best resection and functional results can be achieved.

https://thejns.org/doi/abs/10.3171/2019.5.JNS19453

KEYWORDS gliomas; clinical outcome; intraoperative mapping; neurophysiology; functional balance; extent of resection; morbidity; deficit; oncology

ABBREVIATIONS AED = antiepileptic drug; $c M T$ = cortical motor threshold; DWI = diffusion-weighted imaging; ECoG = electrocorticography; EEG = electroencephalography; EMG = electromyography; EOR = extent of resection; HF = high frequency; HGG = high-grade glioma; LGG = low-grade glioma; MEP = motor-evoked potential; MRC = Medical Research Council; MT = motor threshold; sMT = subcortical motor threshold; To2 = train of 2; To5 = train of 5 .

SUBMITTED February 25, 2019. ACCEPTED May 8, 2019.

INCLUDE WHEN CITING Published online August 9, 2019; DOI: 10.3171/2019.5.JNS19453.

${ }^{*}$ M.R. and M.C.N. share first authorship of this work. 
$\mathrm{R}$ ESECTION of tumors in the primary motor cortex (M1) is usually considered a surgical off-limits area due to its essential role in motor function. ${ }^{6,12}$ It requires the identification of critical motor sites during surgery to maximize tumor resection while maintaining the patient's functional motor integrity., ${ }^{46,19}$ In this regard, the use of intraoperative neurophysiology is the most efficient tool currently available as it offers different stimulation paradigms and probes to be able to locate key motor sites during tumor removal. ${ }^{5,15}$

Despite these advances, resection of tumors harbored primarily in M1 is still highly challenging. M1 is particularly vulnerable to surgical insults. Damage to M1 is usually associated with the occurrence of permanent motor deficits, with limited ability to subsequently recover. ${ }^{8}$ This has historically constrained the surgical approach for M1 tumors to a simple biopsy, eliminating more widespread tumor removal because of consistent and unacceptable long-term morbidity. ${ }^{6}$ Data on surgical treatment of M1 tumors are thus very limited. Some reports are available on limited series or anecdotal cases, mainly involving well-demarcated tumors, ${ }^{11}$ in which traditionally available mapping paradigms are, to a certain extent, quite efficient. Studies in larger series on different tumor types reported variable extent of resection (EOR) and postoperative permanent morbidity.,10,17,18 More recently, evidence that the assistance of a brain mapping technique applied to M1 tumors affords an excellent resection suggests that these tumors are indeed amenable to resection and should not be labeled unresectable., ${ }^{3,9}$ Resectability and maintenance of patient integrity depend on the ability to successfully identify motor tracts during resection. ${ }^{7}$ In this regard, the choice of the most appropriate neurophysiological protocol to perform motor mapping is critical. ${ }^{3}$ Ideally, the best neurophysiological paradigm should be highly feasible in the operating room, should work in all clinical conditions, and should be associated with the lowest percentage of false-negative or false-positive results. Various paradigms or probes are currently available for motor mapping, and their properties, clinical use, and pitfalls have been described. ${ }^{20,21}$ In a previous study reviewing the use of available neurophysiological protocols for guiding resection of tumors involving motor pathways, high-frequency (HF) stimulation (pulse technique) emerged as the most efficient tool to stimulate the motor pathways because of its flexibility of use in different clinical settings, allowing a high percentage of resection and a low rate of deficits. ${ }^{3}$ However, few data are available on the use of HF stimulation for removal of tumors predominantly involving M1. In recent years we have routinely submitted patients harboring tumors within M1 to surgery, moving toward the idea that resection can be performed efficiently and that it is beneficial for both oncological and functional reasons. In all cases, HF stimulation was used as a guide for performing both cortical and subcortical mapping.

In this study we retrospectively analyzed a series of 102 patients with brain tumors admitted between 2013 and 2018 for whom more than $75 \%$ of the tumor mass was harbored within M1, and critically reviewed the use of HF stimulation as a guide. The neurophysiological mapping protocols and/or probes adopted were described and cor- related with patients' clinical features or tumor imaging characteristics. Feasibility of mapping, EOR, and motor function assessment were used to evaluate the oncological and functional outcome to be correlated with the selected neurophysiological parameters used for guiding resection. The aim was to define the most efficient neurophysiological protocol to guide resection for each clinical condition.

\section{Methods \\ Patients}

We reviewed patients admitted to our service (Neurosurgical Oncology Unit, Humanitas Research Hospital) between May 2013 and April 2018. Only patients with $75 \%$ or more of the tumor mass harbored within M1 were included, as determined on axial FLAIR images for lowgrade gliomas (LGGs) and on axial T1-weighted postgadolinium images for high-grade gliomas (HGGs). All patients provided informed consent for the procedure, covered by our Ethical Committee.

\section{Imaging}

The preoperative MRI protocol, acquired on a Siemens Magnetom Verio 3.0-T machine, included 1) axial 3D FLAIR imaging; 2) post-Gd 3D T1-weighted imaging; and 3) diffusion-weighted imaging (DWI) and apparent diffusion coefficient DWI. Patients underwent both immediate (within 48 hours) and 2-month postoperative MRI (volumetric FLAIR and post-Gd T1-weighted) to estimate EOR. ${ }^{22}$ Immediate postoperative diffusion-weighted MRI scans were performed to evaluate ischemia.

\section{Surgical Procedure}

The aim of resection was a complete resection when feasible, according to functional boundaries. With the aid of neuronavigation, a craniotomy tailored to expose the cortex corresponding to the tumor area and a limited amount of surrounding tissue was performed. Cortical mapping was performed to define the cortical safe-entry zone. Subcortical brain mapping was then started, working at the tumor periphery to locate functional boundaries since the initiation of the resection. The mass was finally removed only when subcortical tracts were identified and the tumor was functionally disconnected. For pure rolandic tumors, the peripheral functional motor borders were initially located all around the tumor, and then the tumor mass was removed. In cases of tumors extending anteriorly or posteriorly, subcortical functional tumor disconnection was started at the M1 level and continued anteriorly (in pre-M1 tumors) or posteriorly (in post-M1 tumors). All surgeries were performed by the senior author (L.B.) along with the first authors (M.R. and M.C.N.).

\section{Neurophysiological Protocols}

The intraoperative neurophysiology protocol used to guide resection consisted of brain monitoring and mapping tools.

\section{Brain Monitoring}

Electroencephalography (EEG) and electrocorticogra- 
phy (ECoG) were continuously monitored to assess the depth of anesthesia and occurrence of seizures/afterdischarges. Free-running electromyography (EMG) activity was monitored from the beginning with a multichannel recording setup; as many as 24 muscles were recorded from different contralateral and ipsilateral muscles. The recording system was aimed at monitoring the following during surgery: 1) free-running background EMG activity; 2) motor responses to brain mapping stimulation; and 3) motor-evoked potentials (MEPs) evoked by stimulation of M1 using the "train of 5" (To5) technique throughout the procedure to monitor the integrity of descending motor pathways. MEPs were recorded from transcranial electrodes from incision to closure, and from strip electrodes during the resection period (from dura opening to dura closure). ${ }^{3}$ Small 4 -contact strips were placed very close to the surgical field; occasionally, cortical MEPs were also recorded during resection by direct cortical stimulation to warrant corticofugal fiber integrity. Both contralateral and ipsilateral orofacial as well as upper and lower limb muscle responses were recorded. ${ }^{3}$

\section{Brain Mapping}

Mapping techniques were used to perform cortical mapping and to locate functional boundaries subcortically. HF constant-current stimulation was adopted. Stimulation paradigms consisted of a short train of stimuli (2-9), with variable pulse duration (500-800 $\mu \mathrm{sec})$ and interstimulus interval (2-4 msec), which were modified according to the clinical context. Stimulation was normally delivered using a monopolar probe; however, stimulation with a bipolar probe was also adopted in conditions in which increased focality was needed. Anodal polarity was used for cortical stimulation and cathodal for subcortical stimulation. The reference electrode was placed very close to M1 at the mesial border of the craniotomy. ${ }^{3}$ Initially, a To5 pulse was delivered at either the cortical or subcortical level. According to the clinical context, the pulses were decreased to either 1 or a train of 1 or 2 , or increased to trains of 7 or 9 ; in the latter cases, pulse duration could also be increased to 800 $\mu$ sec. In these cases, current intensity was not increased above $25 \mathrm{~mA}$. For cortical mapping, when a stable motor response was obtained, the intensity of cortical stimulation (mA) was progressively decreased to threshold (cortical motor threshold [cMT]) in order to find the motor hotspot. Hence, a strip electrode was placed for MEP monitoring. For subcortical mapping, intensity of stimulation was used to appreciate distance from the corticofugal pathways, and progressively decreased to threshold (subcortical motor threshold [sMT]) to define the margins of resection. Both contralateral and ipsilateral orofacial as well as upper and lower limb muscles were recorded. ${ }^{3}$

\section{Factors Considered for Analysis}

Demographic and clinical features at admission included age, sex, clinical history and presenting symptoms, previous treatments, type and number of seizures and seizure control, and current and previous drug uptake. Neurological assessment was performed at admission, at 1 week after surgery, and at a 1-month follow-up. Motor conditions were categorized using the Medical Research Council
(MRC) scale evaluation, and requirement for rehabilitation was recorded.

Tumor imaging variables were deducible from conventional preoperative MRI and included volume, zone location, side, border, presence of contrast enhancement, outcrop of the M1 cortex, and extension outside the M1 cortex. Tumor volume was calculated using Smartbrush software (Elements, BrainLAB); for contrast-enhancing lesions, the contrast-enhancing portion of the tumor (the target of resection) was measured. For low-grade, noncontrast-enhancing tumors, the FLAIR signal (the target of resection) was used. To evaluate tumor location, M1 was divided into three motor zones (zone 1, lower limb; zone 2 , upper limb; and zone 3 , face), as previously reported. ${ }^{9}$ Tumor borders were defined as well defined or irregular, 1,2 using postcontrast imaging for contrast lesions, or FLAIR images for those that were nonenhancing. Outcropping of M1 was defined as present when the tumor was reaching the cortex, either on coronal and/or sagittal FLAIR images for nonenhancing lesions, or on T1-weighted postcontrast images for enhancing lesions. Regarding the extension of the tumor, only tumors with more than $75 \%$ of their mass involving M1 were included in the present series; in cases of large tumor volume, the tumor could extend anteriorly toward the dorsal premotor cortex or the supplementary motor cortex or posteriorly toward the primary sensory cortex. The extension was categorized accordingly.

Tumor histological variables included histology and molecular profile (IDH1 status, codeletion).

Surgical variables included duration of surgery, mapping features, occurrence of intraoperative complications (bleeding/ischemia/infection), intraoperative mortality, type of anesthesia (asleep vs awake), and EOR. Mapping features considered for the analysis were the mapping protocol used at the cortical or subcortical level, the type of probe used, and the findings during cortical or subcortical mapping; the lowest current intensity threshold reached at the cMT or SMT level; and changes in MEPs recorded with To5 monitoring ( $>50 \%$ reduction of MEP amplitude, and MEP loss).

EOR calculation was performed on immediate postoperative MRI (within 48 hours), targeting postcontrast MRI for enhancing lesions or FLAIR for nonenhancing lesions. EOR corresponded to the percentage of volume resected with respect to the preoperative volume: (preoperative volume - postoperative volume)/preoperative volume. Resection was categorized as 1) total resection (postoperative volume $=0 \mathrm{~cm}^{3}$ ), 2) subtotal resection (postoperative volume between 1 and $\mathrm{cm}^{3}$, or 3 ) partial resection (postoperative volume $>5 \mathrm{~cm}^{3}$ ).

Postoperative diffusion-weighted MRI scans were also performed to rule out ischemia, and the amount of DWI abnormalities was categorized as previously reported. ${ }^{9}$ All radiological features considered for the analysis were evaluated by the first authors (M.R. and M.C.N.) and senior author (L.B.)

\section{Statistical Analysis}

All statistical analyses were performed using IBM SPSS software (IBM Corp.). For categorical data, chisquare (multiple categories) or Fisher exact (2-category) 
tests were reported. Univariate logistic regression was performed and used to calculate odds ratios. Two-tailed t-tests were used for comparisons between continuous variables when comparing two variables. ANOVA was used for comparisons between continuous variables when comparing three or more variables.

\section{Results}

\section{Patients}

One hundred two patients with a tumor principally involving M1 were treated surgically. Clinical and imaging features are reported in Table 1. Most patients experienced seizures, requiring at least 1 antiepileptic drug (AED) to be controlled. Poor seizure control was observed in $39.2 \%$ of cases. Most of the tumors had a small volume, were located prevalently in Berger zones 1 and 2, and in half of the cases reached the surface. Tumor borders were irregular in 78 cases $(76.5 \%)$ and in $39(38.2 \%)$ contrast enhancement was present. According to the inclusion criteria, the largest proportion of the tumor bulk $(>75 \%)$ was located within M1; extension outside M1 was documented in $20.6 \%$ of cases only, either anteriorly (12 cases) or posteriorly (9 cases; Fig. 1).

\section{Surgical Data and EOR}

Surgery was always performed under general anesthesia. EEG and ECOG were used to monitor the level of anesthesia, titrated to obtain continuous EEG and ECOG activity, avoiding any burst suppression. Cortical and subcortical motor mapping was always performed using HF stimulation. Cortical motor mapping was used to define the safe cortical entry zone, identified in all cases; subcortical motor mapping defined the functional boundaries and margins of resection. MEP monitoring, recorded from strip electrodes during the entire resection procedure, showed no abnormalities except in 2 cases (those patients who experienced permanent deficits). No intraoperative seizures were recorded. The mean $( \pm \mathrm{SD})$ duration of surgery was $142 \pm 12$ minutes. Mean duration of cortical or subcortical mapping was $12 \pm 3$ minutes and $14 \pm 2$ minutes, respectively. EOR was total in 87 cases $(85.3 \%)$, subtotal in 7 (6.9\%), and partial in the remaining 8 (7.8\%). Mean EOR was $95.62 \%$. In cases of subtotal and partial removal, mean and median EOR were $70.3 \%$ and $70.1 \%$, respectively. Complications (bleeding) were observed in 2 cases (1.96\%). No postoperative mortality was documented. EOR was not affected by clinical factors or most tumor-related factors, deducible from conventional MRI. Irregular borders, large volume, and absence of contrast enhancement were associated with lower odds of achieving a total removal. Histology did not exert any effect, whereas IDH1 mutation was associated with lower odds of achieving a total resection (Table 2).

\section{Motor Functions}

A decline of motor function was observed in 98 patients $(96.1 \%)$ immediately after surgery (66\% mild, MRC grade 4; severe, MRC grade < 4, in 29\%; Table 1), which completely recovered at follow-up in all but 2 cases. In the latter, the motor deficit was mild (MRC grade 4); these 2 patients were operated on for HGG $(2 / 41,4.9 \%)$. No per- manent deficits were registered in patients who underwent operations for LGGs. A total of 35.3\% of patients underwent rehabilitation. Praxis was affected in $2 \%$ of patients. ${ }^{14}$

\section{Neurophysiological Approaches}

Three main approaches were used: a "standard approach," an "increased train approach," and a "reduced train approach" (Table 3).

\section{Standard Approach}

The standard approach consisted of an HF-To5 paradigm delivered by a monopolar probe. This paradigm was used at the cortical level to define the safe-entry zone, and at the subcortical level to identify motor functional boundaries by stopping the resection when an SMT of $3 \mathrm{~mA}$ was reached, in the presence of a stable MEP recording. This protocol was applied in 51 patients. Regarding presenting symptoms and duration, most patients were diagnosed because of seizures, were neurologically intact at admission, and were taking AEDs with good seizure control in the majority of cases. At imaging, most tumors reached the surface of M1, showing contrast enhancement and a welldefined border in more than $50 \%$ of cases. Tumors were located in all M1 regions. At surgery, using cortical mapping a safe cortical entry zone was identified in all cases, coincident with the outcrop area of the tumor when present. At the subcortical level, a 3-mA motor threshold (MT) was reached in the absence of any alteration in MEP recording parameters. A complete resection was achieved in $72.5 \%$ of cases, a subtotal resection in $11.7 \%$, and a partial in $15.8 \%$. A transient motor decline was observed in the majority of patients during the first week, and all recovered within 1 month except 1 patient (2\%). Motor rehabilitation was needed in $27.5 \%$ of cases. This mapping approach was applied for resection of tumors that were low grade, high grade, and metastasized. When considering the 14 cases of partial/subtotal resection, it was found that the majority were characterized by large, irregular border tumors, with no contrast enhancement; histologically, these tumors were LGGs. In contrast, a total resection was achieved in patients with tumors characterized mostly by contrast enhancement and well-defined borders, in most cases diagnosed at histological analysis as HGGs (Table 3).

\section{Increased Train Approach}

The increased train approach consisted of an increase in the number of train pulses (7 or 9) and of the pulse duration $(800 \mu \mathrm{sec}$ instead of $500 \mu \mathrm{sec})$, delivered by a monopolar probe. This approach was applied when, at a cortical level, the standard To5 approach failed to evoke a motor response even at a high current intensity $(25 \mathrm{~mA})$, preventing the acquisition of a cortical map, resulting in a complete negative cortical mapping. This occurred in 8 patients (7.8\%). A train of 7 was used in 2 patients, a train of 9 in the remaining patients. The pulse duration was increased to 800 usec in 5 cases. In 3 cases, the reversal of stimulation polarity (from anodal to cathodal) was needed to obtain reliable responses at the cortical level, or to obtain responses with a lower current intensity. By using this approach, sites evoking MEPs were detected, allowing for a reliable cortical mapping and identification of a safe cortical area in which to perform the corticectomy and initiate 
TABLE 1. Demographic, clinical, radiological, and surgical features of all patients included in the study $(n=102)$

\begin{tabular}{|c|c|c|}
\hline Variable & Value & $\%$ \\
\hline \multicolumn{3}{|l|}{ Clinical and demographic features } \\
\hline \multicolumn{3}{|l|}{ Sex } \\
\hline Male & 62 & 60.8 \\
\hline Female & 40 & 39.2 \\
\hline \multicolumn{3}{|l|}{ Age, yrs } \\
\hline Mean & 42.7 & \\
\hline Range & $17.2-75$ & \\
\hline \multicolumn{3}{|l|}{ Clinical onset } \\
\hline Focal seizure & 91 & 89.2 \\
\hline Other & 11 & 10.8 \\
\hline Duration of clinical $\mathrm{Hx}>6$ mos & 62 & 60.8 \\
\hline \multicolumn{3}{|l|}{ No. of AEDs before surgery } \\
\hline 1 & 44 & 43.1 \\
\hline$>1$ & 58 & 56.9 \\
\hline Controlled preop seizures & 62 & 60.8 \\
\hline Pts w/ preop motor deficit & 10 & 9.8 \\
\hline \multicolumn{3}{|l|}{ Preop MRC scale score } \\
\hline 5 & 92 & 90.2 \\
\hline 4 & 10 & 9.8 \\
\hline \multicolumn{3}{|l|}{ Radiological features } \\
\hline \multicolumn{3}{|l|}{ Side } \\
\hline $\mathrm{Lt}$ & 35 & 34.3 \\
\hline Rt & 67 & 65.7 \\
\hline \multicolumn{3}{|l|}{ Tumor volume, $\mathrm{cm}^{3}$} \\
\hline Mean & 10.13 & \\
\hline Median & 5.6 & \\
\hline Range & $1.3-65$ & \\
\hline \multicolumn{3}{|l|}{ Contrast-enhancing lesion } \\
\hline Yes & 39 & 38.2 \\
\hline No & 63 & 61.8 \\
\hline \multicolumn{3}{|c|}{$\begin{array}{l}\text { Tumor motor zone (Berger classifica- } \\
\text { tion)* }\end{array}$} \\
\hline 1 & 40 & 39.2 \\
\hline 2 & 54 & 52.9 \\
\hline 3 & 8 & 7.8 \\
\hline \multicolumn{3}{|l|}{ Tumor extension } \\
\hline No & 81 & 79.4 \\
\hline Anterior & 12 & 11.8 \\
\hline Posterior & 9 & 8.8 \\
\hline Cortical outcrop & 80 & 78.4 \\
\hline \multicolumn{3}{|l|}{ Border } \\
\hline Highly irregular & 78 & 76.5 \\
\hline Well defined & 24 & 23.5 \\
\hline \multicolumn{3}{|l|}{ Postop tumor volume, $\mathrm{cm}^{3}$} \\
\hline Mean & 1.06 & \\
\hline Median & 0 & \\
\hline Range & $0-29$ & \\
\hline
\end{tabular}

CONTINUED IN NEXT COLUMN »
» CONTINUED FROM PREVIOUS COLUMN

TABLE 1. Demographic, clinical, radiological, and surgical features of all patients included in the study $(n=102)$

\begin{tabular}{lcc}
\hline \multicolumn{1}{c}{ Variable } & Value & $\%$ \\
\hline Radiological features (continued) & & \\
\hline Resection & & \\
\hline Partial (residual tumor $>5 \mathrm{ml}$ ) & 8 & 7.8 \\
\hline Subtotal (residual tumor $=1-5 \mathrm{ml})$ & 7 & 6.9 \\
\hline Total (residual tumor $=0 \mathrm{ml}$ ) & 87 & 85.3 \\
\hline EOR & & \\
\hline Mean & 95.62 & \\
\hline $100 \%$ & 87 & 85.3 \\
\hline $90-99 \%$ & 2 & 2 \\
\hline$<90 \%$ & 13 & 12.7 \\
\hline DWI alteration & & \\
\hline Yes & 6 & 5.9 \\
\hline No & 92 & 90.2 \\
\hline NA & 4 & 3.9 \\
\hline
\end{tabular}

Surgical features \& outcome

Neurophysiological approach

\begin{tabular}{lrc} 
Standard & 51 & \multicolumn{1}{c}{50} \\
\hline Reduced train & 43 & 42.2 \\
Increased train & 8 & 7.8 \\
Worsening of motor deficit & & \\
1 wk & 98 & 96.1 \\
1 mo & 2 & 2
\end{tabular}

1-wk MRC scale

\begin{tabular}{lcc}
\hline 5 & 4 & 3.9 \\
\hline 4 & 68 & 66.7 \\
\hline 1-mo MRC scale & 30 & 29.4 \\
\hline 5 & & \\
\hline 4 & 100 & 98 \\
\hline Postop apraxia & 2 & 2 \\
\hline Postop rehabilitation & 2 & 2 \\
\hline Histology & 36 & 35.3 \\
\hline LGG & & \\
\hline HGG & 54 & 52.9 \\
\hline Other & 41 & 40.2 \\
\hline IDH1-2 & $7 \dagger$ & 6.9 \\
\hline Wildtype & & \\
\hline Mutated & 45 & 44.1 \\
\hline NA & 50 & 49 \\
\hline 1p/19 codeletion & 7 & 6.9 \\
\hline Yes & & \\
\hline NA & 19 & 18.6 \\
\hline
\end{tabular}

$\mathrm{Hx}=$ history $\mathrm{NA}=$ not available; pts = patients.

* Classification of zones of the motor cortex based on Berger: zone $1=$ lower limb, zone 2 = upper limb, zone 3 = face.

† Other $=7$ metastases ( 6 lung, 1 breast). 

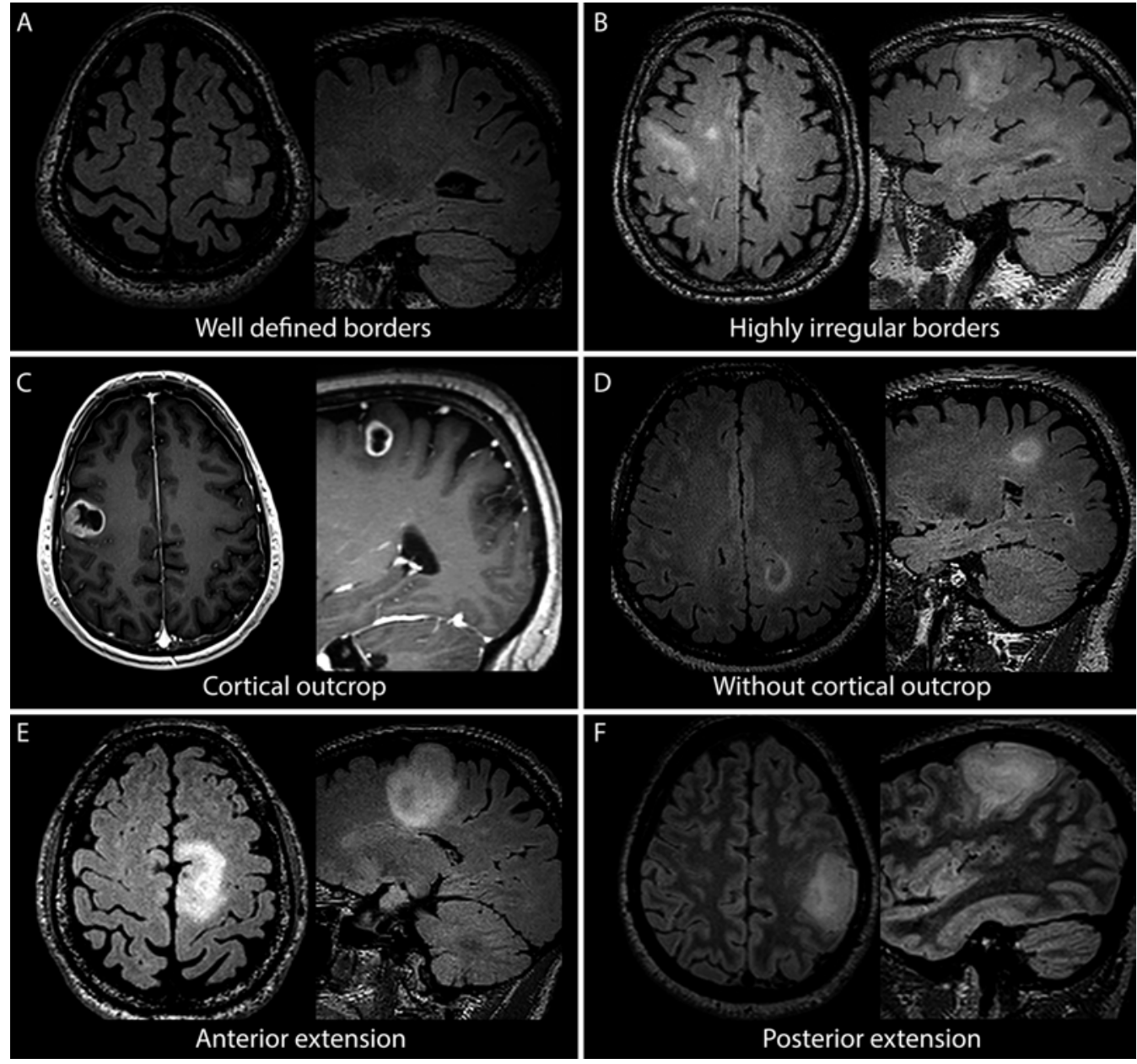

FIG. 1. Example cases of the radiological features considered on preoperative MRI. Upper row: A case of LGG in the left zone 2 with well-defined borders (A), and a case of LGG in the right zone 2 with highly irregular borders (B). Middle row: A case of HGG in the right zone 2 with cortical outcrop (C; the superior border of the tumor reached the cortical surface), and a case of LGG in the left zone 1 without cortical outcrop (D; the tumor did not reach the cortical surface). Lower row: A case of LGG in the left zone 1 with an anterior extension toward the supplementary motor area (E), and a case of LGG in the left zone 2 with a posterior extension toward the sensory cortex $(\mathbf{F})$.

the resection in all patients. These were complex patients with a long clinical history $-75 \%$ received previous treatment (radiosurgery or surgery with radio-chemotherapy; Table 3) - who presented with poorly controlled seizures despite the high number of AEDs, and already had mild motor deficits at admission (Table 3). This same increased train approach was used at the subcortical level to guide resection (Fig. 2). In all cases, the train was reduced to a To5 at the end of resection, allowing for complete tumor removal (sMT of $5 \mathrm{~mA}$ ). In these patients MEP monitoring from the strip electrodes also required the use of an increased train, and only at the end of the resection did the standard To5 paradigm regain efficacy. EOR was complete in $87.5 \%$ of cases and subtotal in the only case remaining. No motor deficits were recorded at 1 month. Histology revealed recurrent high-grade tumors or metastases (Table 3).

\section{Reduced Train Approach}

The reduced train approach consisted of the parallel and combined use of train of 2 (To2) pulses (sometimes only 1 pulse), associated with the standard To5 paradigm, to perform the cortical and subcortical mapping and to define the margins of resection. Both paradigms were delivered using a monopolar probe and applied in 43 cases (42.5\%), mostly in the last 3 years. Patients in this group had a long clinical history; $50 \%$ of cases showed poorly controlled seizures despite a large number of AEDs at high doses. All but 2 patients were neurologically intact at admission. They were harboring tumors located in all M1 regions, characterized by highly irregular margins and no contrast enhancement, resembling LGGs at standard clinical imaging. At least $40 \%$ of these cases did not reach the cortical surface. This approach consisted first of cortical mapping to identify the negative safe-entry zone, followed by subcortical mapping to define the margins of resection. During mapping for both paradigms in a single site, at either the cortical or subcortical level, the MT was always identified specific to the paradigm. Regarding the cortical mapping, the standard To5 paradigm, when applied to these patients at cMT, elicited MEPs (often from several muscles) 
TABLE 2. Variables related to residual tumor

\begin{tabular}{|c|c|c|c|c|}
\hline \multirow[b]{2}{*}{ Variable } & \multirow{2}{*}{$\begin{array}{c}\text { No. } \\
\text { of } \\
\text { Pts }\end{array}$} & \multicolumn{2}{|l|}{ EOR } & \multirow[b]{2}{*}{$\begin{array}{c}\mathrm{p} \\
\text { Value }^{*}\end{array}$} \\
\hline & & $\begin{array}{c}\text { Partial/Subtotal } \\
(\%)\end{array}$ & $\begin{array}{l}\text { Total } \\
(\%)\end{array}$ & \\
\hline Contrast enhancement & & & & 0.004 \\
\hline Yes & 39 & $2(5.1)$ & $37(94.9)$ & \\
\hline No & 63 & $18(28.6)$ & $45(71.4)$ & \\
\hline Extension outside M1 & & & & 0.000 \\
\hline No & 82 & $10(12.2)$ & $72(87.8)$ & \\
\hline Yes & 20 & $10(50)$ & $10(50)$ & \\
\hline M1 outcrop & & & & 0.045 \\
\hline No & 22 & $1(4.5)$ & $21(95.5)$ & \\
\hline Yes & 80 & $19(23.8)$ & $61(76.2)$ & \\
\hline Borders & & & & 0.029 \\
\hline Well defined & 24 & $1(4.2)$ & $23(95.8)$ & \\
\hline Highly irregular & 78 & $19(24.4)$ & $59(75.6)$ & \\
\hline Preop mean volume, $\mathrm{cm}^{3}$ & & 21.57 & 7.03 & 0.000 \\
\hline IDH1 status & & & & 0.027 \\
\hline Mutated & 50 & $15(30)$ & $35(70)$ & \\
\hline Wildtype & 45 & $5(11.1)$ & $40(88.9)$ & \\
\hline Other & 7 & $0(0)$ & $7(100)$ & \\
\hline Rehabilitation & & & & 0.002 \\
\hline No & 66 & $7(10.6)$ & $59(89.4)$ & \\
\hline Yes & 36 & $13(36.1)$ & $23(63.9)$ & \\
\hline Duration clinical $\mathrm{Hx}$, mos & & & & 0.346 \\
\hline$>6$ & 62 & $14(22.6)$ & $48(77.4)$ & \\
\hline$<6$ & 40 & $6(15)$ & $34(85)$ & \\
\hline Previous treatment & & & & 0.856 \\
\hline Yes & 39 & $8(20.5)$ & $31(79.5)$ & \\
\hline No & 63 & $12(19)$ & $51(81)$ & \\
\hline Side & & & & 0.262 \\
\hline Rt & 67 & $11(16.4)$ & $56(83.6)$ & \\
\hline $\mathrm{Lt}$ & 35 & $9(25.7)$ & $26(74.3)$ & \\
\hline Motor zone & & & & 0.280 \\
\hline 1 & 40 & $7(17.5)$ & $33(82.5)$ & \\
\hline 2 & 54 & $11(20.4)$ & $43(79.6)$ & \\
\hline 3 & 8 & $2(25)$ & $6(75)$ & \\
\hline Preop motor deficit & & & & 0.383 \\
\hline No & 92 & $17(18.5)$ & $75(81.5)$ & \\
\hline Yes & 10 & $3(30)$ & $7(70)$ & \\
\hline Histomolecular diagnosis & & & & 0.278 \\
\hline LGG & 54 & $13(24.1)$ & $41(75.9)$ & \\
\hline HGG & 41 & $7(17.1)$ & $34(82.9)$ & \\
\hline Other & 7 & $0(0)$ & $7(100)$ & \\
\hline
\end{tabular}

Boldface type indicates statistical significance.

* A Pearson chi-square test for the percentage of patients in each resection group (total resection in comparison to subtotal/partial resection) was performed for all variables, with the exception of preoperative tumor volume, in which an ANOVA test was used.
TABLE 3. Differences between the 3 groups according to neurophysiological approach

\begin{tabular}{|c|c|c|c|c|}
\hline \multirow[b]{2}{*}{ Variable } & \multicolumn{3}{|c|}{ Neuropsychological Approach (\%) } & \multirow[b]{2}{*}{$\begin{array}{c}p \\
\text { Value }^{*}\end{array}$} \\
\hline & $\begin{array}{l}\text { Standard } \\
(n=51)\end{array}$ & $\begin{array}{c}\text { Reduced } \\
(n=43)\end{array}$ & $\begin{array}{c}\text { Increased } \\
(n=8)\end{array}$ & \\
\hline Previous treatment & & & & 0.039 \\
\hline Yes & $15(29.4)$ & $18(41.9)$ & $6(75)$ & \\
\hline No & $36(70.6)$ & $25(58.1)$ & $2(25)$ & \\
\hline Preop deficit & & & & 0.000 \\
\hline Yes & $1(2)$ & $5(11.6)$ & $4(50)$ & \\
\hline No & $50(98)$ & $38(88.4)$ & $4(50)$ & \\
\hline Histomolecular diagnosis & & & & 0.000 \\
\hline LGG & $23(45.1)$ & $31(72.1)$ & $0(0)$ & \\
\hline HGG† & $28(54.9)$ & $12(27.9)$ & $8(100)$ & \\
\hline EOR & & & & 0.136 \\
\hline Total & $37(72.5)$ & $38(88.4)$ & $7(87.5)$ & \\
\hline Subtotal/partial & $14(27.5)$ & $5(11.6)$ & $1(12.5)$ & \\
\hline Postop deficit (1 wk) & & & & 0.566 \\
\hline Yes & $48(94.1)$ & $42(97.7)$ & $8(100)$ & \\
\hline No & $3(5.9)$ & $1(2.3)$ & $0(0)$ & \\
\hline Postop deficit (1 mo) & & & & 0.909 \\
\hline Yes & $1(2)$ & $1(2.3)$ & $0(0)$ & \\
\hline No & $50(98)$ & $42(97.7)$ & $8(100)$ & \\
\hline Rehabilitation & & & & 0.237 \\
\hline Yes & $14(27.5)$ & $19(44.2)$ & $3(37.5)$ & \\
\hline No & $37(72.5)$ & $24(55.8)$ & $5(62.5)$ & \\
\hline
\end{tabular}

Boldface type indicates statistical significance.

* The Pearson chi-square test was used for all variables.

† Includes 7 "other" histological diagnoses (7 metastases).

in every investigated site, preventing the identification of a safe (negative) entry zone in which to perform the corticectomy and start the resection (Fig. 3). When the mapping was repeated with the To 2 in the same cortical sites at cMT, in some sites MEPs were not evoked, allowing the identification of a negative safe-entry zone. This approach was particularly useful in cases of tumors not reaching the M1 surface (Fig. 3B). Regarding the subcortical mapping, the strategy was to continue with the resection until the lowest sMT was reached. For both paradigms, the lowest current intensity to define the sMT was $2 \mathrm{~mA}$. In this scenario, typically we started subcortical mapping with the To5 paradigm, defining the sMT in each site. The resection was continued until an sMT with a current intensity of 2 $\mathrm{mA}$ was identified, detecting MEPs from several muscles. Then, in the same site, we shifted to the To2, and either no MEPs were evoked, or MEPs from fewer muscles, usually at higher current intensity $(8-10 \mathrm{~mA})$, were elicited. This allowed us to continue with the resection until an SMT of $2 \mathrm{~mA}$ was reached, ultimately using this sMT to define the functional boundaries and the margin of resection, in the presence of stable MEP recording (performed with the standard To5 technique from the strip electrodes; Fig. 4). In 12 cases (27.9\%), a bipolar probe was used to increase the spatial resolution of the mapping, despite requiring 

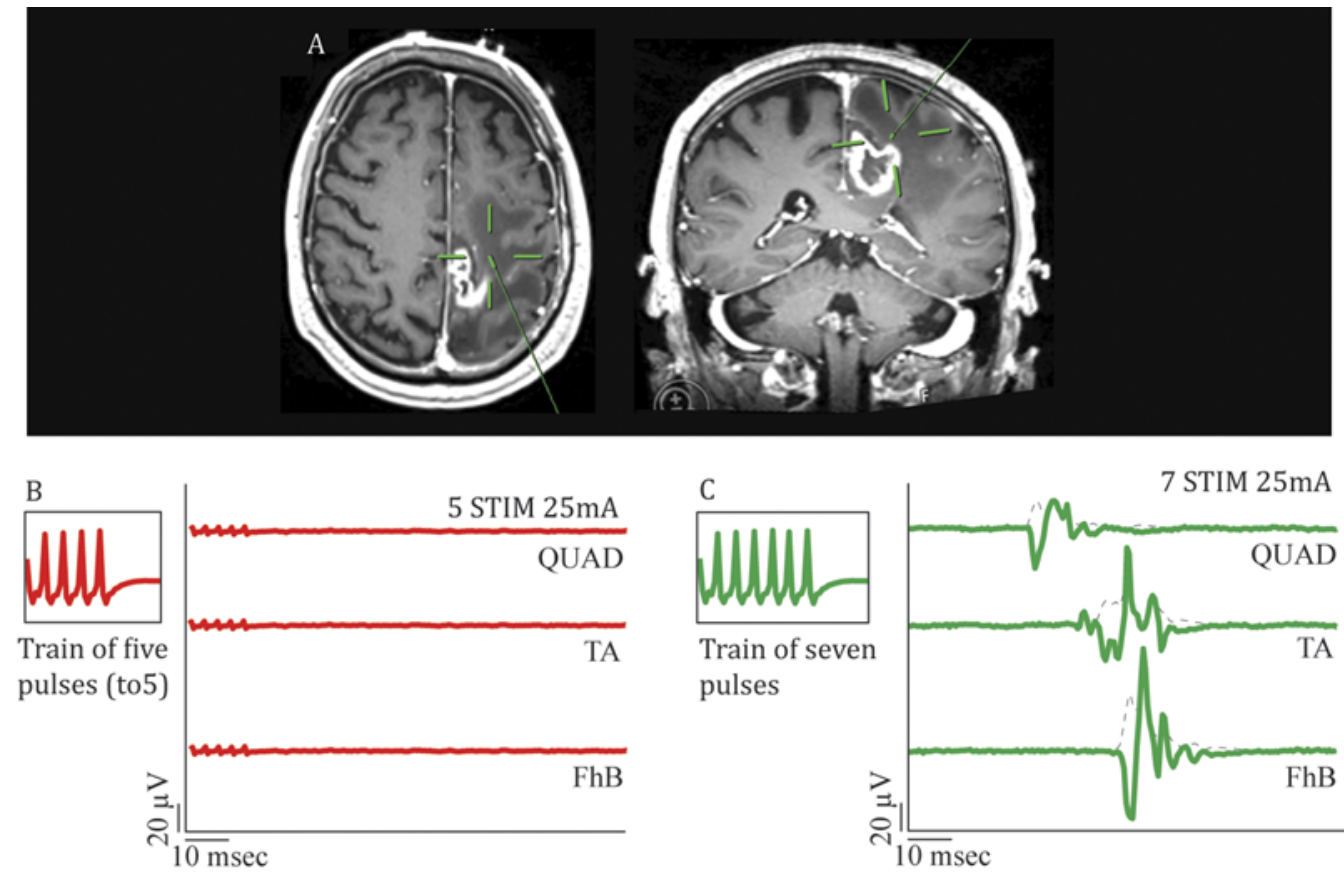

FIG. 2. Example of a tailored neurophysiological approach: the increased train approach. This example involved a left metastasis located in zone 1 previously treated with radiosurgery (panel A: postcontrast T1-weighted images, axial and coronal). The increased train approach was used for performing the cortical and subcortical mapping. When the standard To5 paradigm was used at the subcortical level for guiding resection, it failed in evoking any MEPs (panel B; $20 \mu \mathrm{V}$ per division) even when applied at high current intensity $(25 \mathrm{~mA}$ ), while the increased train approach (train of 7 , pulse duration $0.5 \mathrm{msec}$ ) evoked MEP responses from several leg muscles (quadriceps femoris [QUAD], tibialis anterior [TA], and flexor hallucis brevis [FhB]; $20 \mu \mathrm{V}$ per division; panel C) at the same current intensity $(25 \mathrm{~mA})$. Figure is available in color online only.

a higher current intensity (4-7 mA), allowing for an extended dissection. Total resection was reached in $88.4 \%$ of cases, and subtotal resection in the remaining patients (Fig. 5). In the first postoperative week $97.7 \%$ of patients experienced a decline in motor function, and all recovered except 1 patient (2.3\%, mild motor deficit), requiring 2 weeks rehabilitation in $44.2 \%$ of cases. Histological analysis diagnosed $72.1 \%$ of these tumors as LGGs (Table 3).

\section{Discussion}

Recent data demonstrated that M1 tumors are amenable to resection with an acceptable low morbidity. ${ }^{9}$ Resection feasibility is strongly associated with the use of a brain mapping technique. ${ }^{5}$ In this regard, the challenge is to find the adequate stimulating protocol to identify functional structures in the largest number of clinical conditions. ${ }^{3}$ The clinical impact of the stimulation paradigm has to be evaluated in terms of feasibility of mapping (number of cases in which a reliable mapping can be obtained) and clinical outcome (EOR and rate of permanent morbidity).

Few clinical data are at present available on the use of a brain mapping technique for guiding resection of M1 tumors, and most of these studies refer to the use of lowfrequency stimulation (Penfield's). ${ }^{7,10,17,18}$ Complete tumor resection was reported in $50.9 \%$ of cases, with the occurrence of permanent deficits in $37.7 \%$, and only $4 \%$ of patients experiencing severe motor deficits. ${ }^{9}$ Resection was performed in low- and high-grade tumors, located in all M1 sites. We previously demonstrated that in resection of tumors affecting motor pathways, the best clinical impact was obtained when the neurophysiological protocol was adapted to the clinical context, defined by patient clinical history and tumor imaging features. ${ }^{3}$ These data suggested that HF stimulation is the most efficient tool for testing motor pathways because of its high versatility in being able to adapt the appropriate cMT, sMT, and probe based on the clinical context encountered at surgery. However, the number of patients with tumors primarily involving $\mathrm{M} 1$ in that series was limited. Since then, we have routinely applied HF stimulation as a guide during resection of tumors within M1. In this study, we reviewed this experience, evaluating the feasibility of mapping, EOR, and motor function assessment, and correlating these features with the chosen neurophysiological parameters guiding resection. The aim was to define the most efficient neurophysiological protocol to guide resection for each clinical condition.

Our data confirmed that resection of tumors principally involving M1 is feasible. The chance of achieving a complete resection was high (85.3\%), particularly in cases of tumors with well-defined borders or contrast enhancement, independent of location or a patient's clinical features. However, a complete resection was also feasible in the large majority of irregular border tumors (lower grade), or in those with previous treatments, when an appropriate neurophysiological approach was applied (Table 3). Although the rate of immediate postoperative deficits was high, as expected when functional boundaries are reached at the subcortical level, resection was associated with low 

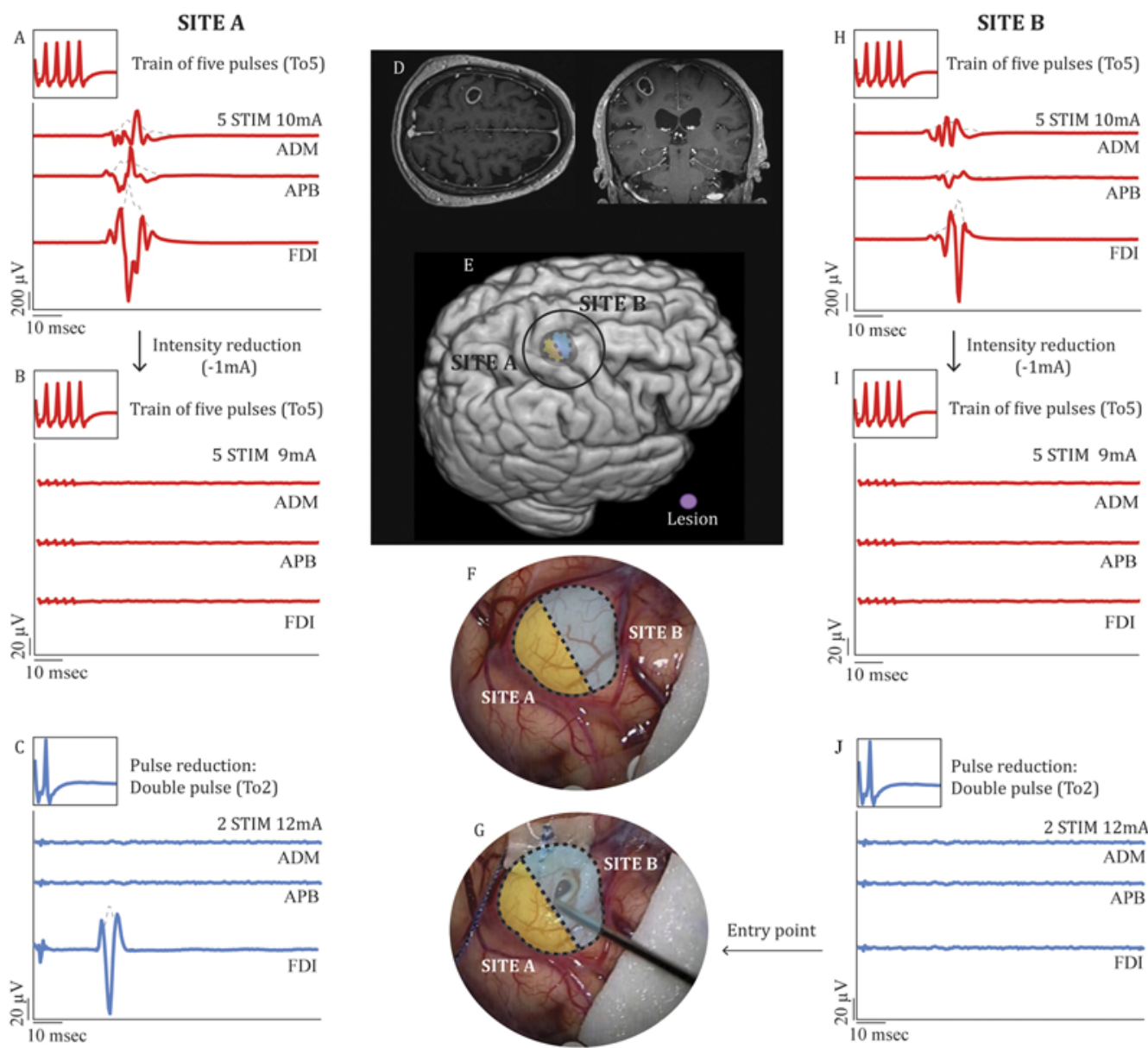

FIG. 3. Example of a tailored neurophysiological approach: the reduced train approach at the cortical level. This patient had an HGG in the right zone 2 (postcontrast T1-weighted imaging, axial and coronal; panel D) in which a reduced train approach was used for performing the cortical mapping and defining the safe cortical entry zone. Surgery was performed under general anesthesia. The standard To5 approach was combined with the reduced train approach at cMT. The mapping was started at the posterior border of the hand knob, shown as a yellow area, named as SITE A in the 3D reconstruction (panel E) of the brain and of the tumor (indicated by a pink oval) and in the picture of the surgical field (panel F). When the To5 paradigm delivered by a monopolar probe was applied to explore this area, it identified MEPs from several muscles at the cMT; the MEPs recorded from a representative site are shown in panel $\mathbf{A}$. MEPs from the abductor digiti minimi (ADM), abductor pollicis brevis (APB), and first dorsal interosseous (FDI; $200 \mu \mathrm{V}$ per division) were obtained at $10 \mathrm{~mA}$ (cMT). As shown by the disappearance of any response when in the same site, the current intensity was decreased to $9 \mathrm{~mA}$ (panel B: ADM, APB, and FDI; $20 \mu \mathrm{V}$ per division). In the site, the reduction of the train to To2 at cMT (12 mA) evoked MEPs from the FDI only (panel C; $20 \mu \mathrm{V}$ per division), confirming SITE A as a "positive" site, and therefore not a site to start with the resection $(20 \mu \mathrm{V}$ per division was used as a scale in the last two images for comparison). Then the mapping was moved to the anterior border of the hand knob, shown as a pale blue area, named as SITE B in the 3D reconstruction of the brain and the tumor (panel E) and in the image of the surgical field (panel F). When the To5 paradigm, delivered by a monopolar probe, was applied in this area at CMT, it evoked MEP responses from several hand muscles. The MEPs recorded in a representative site are shown in panel $\mathrm{H}$ (MEPs from ADM, APB, and FDI; $200 \mu \mathrm{V}$ per division; cMT at 10 $\mathrm{mA}$ ). The reduction of the intensity to $9 \mathrm{~mA}$ did not evoke any MEPs (panel I; ADM, APB, and FDI; $20 \mu \mathrm{V}$ per division), confirming the achievement of the cMT. In the same site, the To2 paradigm delivered by a monopolar probe did not elicit any MEP responses (panel J: no MEPs; $20 \mu \mathrm{V}$ per division), resulting in a "negative" site. This area was then used as a safe-entry point in which to perform the corticectomy as showed in panel $\mathbf{G}$ (intraoperative image of the surgical field with the established corticectomy). Figure is available in color online only.

permanent morbidity (2\%). Permanent deficits were restricted to patients with HGG, confirming that HGGs are at major risk of morbidity. ${ }^{9}$ These data confirmed HF stimulation as an efficient tool for guiding resection of M1 tumors. In addition, by looking at the mapping procedures adopted during surgery, the related patients' clinical and imaging features, and the obtained clinical results, specific considerations can be assumed.
First, the standard HF approach (To5 with a monopolar probe) was particularly efficient in patients with well-controlled seizures and harboring tumors with well-defined borders or contrast enhancement, and generally reaching the surface. In this setting, the reliability of cortical mapping was high, allowing us to identify the safe-entry zone and, at a subcortical level, the functional motor boundaries at the lowest MT. 


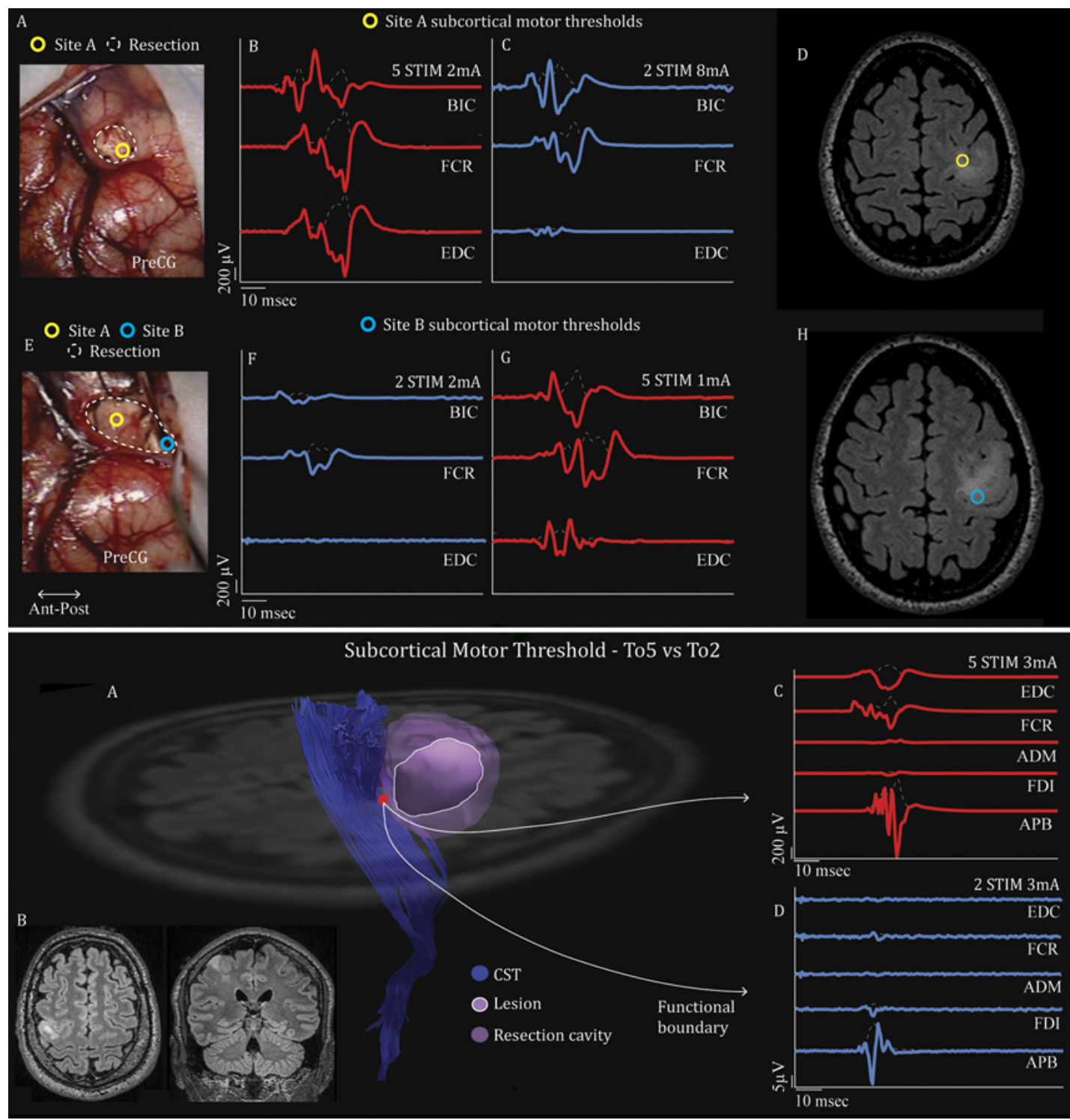

FIG. 4. Example of a tailored neurophysiological approach: the reduced train approach at subcortical level. Upper panel: To guide resection for a dominant zone 2 LGG, during subcortical mapping, the To5 and To2 paradigms were combined. When resection started, To5 delivered by a monopolar probe was applied in a subcortical site (Site A), indicated by a yellow circle at the posterior border of the resection cavity (indicated by a dashed white line in the intraoperative field image; panel A). In this site, the To5 at sMT $(2 \mathrm{~mA}$ ) evoked MEPs from several muscles (biceps [BIC], flexor carpi radialis [FCR], and extensor digitorum communis [EDC]; $200 \mathrm{mV}$ per division; panel B). In the same site, when it was shifted to To2, MEPs were detected from BIC and FCR only (panel C; $200 \mu \mathrm{V}$ per division) at higher sMT (8 $\mathrm{mA}$ ). This allowed us to progress with the resection, continued more inferiorly and posteriorly, using To2 as a guide, until a site (Site B, marked in the intraoperative image of the surgical field as a blue circle; panel D) in which a 2-mA sMT was reached (panel E; MEPs from BIC and FCR; $200 \mu \mathrm{V}$ per division). In this same site, when the T05 was reapplied (panel F) without moving the monopolar probe, MEPs of larger amplitude and from several muscles (BIC, FCR, and EDC) were detected even when the current intensity was decreased to $1 \mathrm{~mA}$ (the lowest allowed by the intraoperative monitoring machine in use (panel G). PreCG = precentral gyrus, where the tumor was located. Panels $\mathbf{D}$ and $\mathbf{H}$ show the location of Site $A$ and Site B as registered during surgery on the preoperative FLAIR images by the neuronavigation system. Lower panel: Use of the reduced train approach at the subcortical level for defining the margin of resection in another case of an LGG located in the right zone 2 (axial and coronal FLAIR images, panel B). The To2 was used at MT in combination with the To5 standard paradigm. While the standard To5 paradigm at MT evoked MEPs from several hand muscles (EDC, FCR, ADM, FDI, and APB; $200 \mu \mathrm{V}$ per division; panel C), the To2 paradigm evoked MEPs from a single muscle (APB; $5 \mu \mathrm{V}$ per division; panel D), increasing the spatial resolution of the stimulation, and allowing us to extend the dissection outside of the tumor area within the M1 descending fibers. Panel A shows the tumor volume as a solid purple volume, the resection cavity as a faint purple volume, the high angular resolution diffusion imaging reconstruction of M1 efferent fibers from the hand knob region (in light blue), and the site where the stimulation induced the previous responses and that served as the margin of resection (red dot). Figure is available in color online only. 

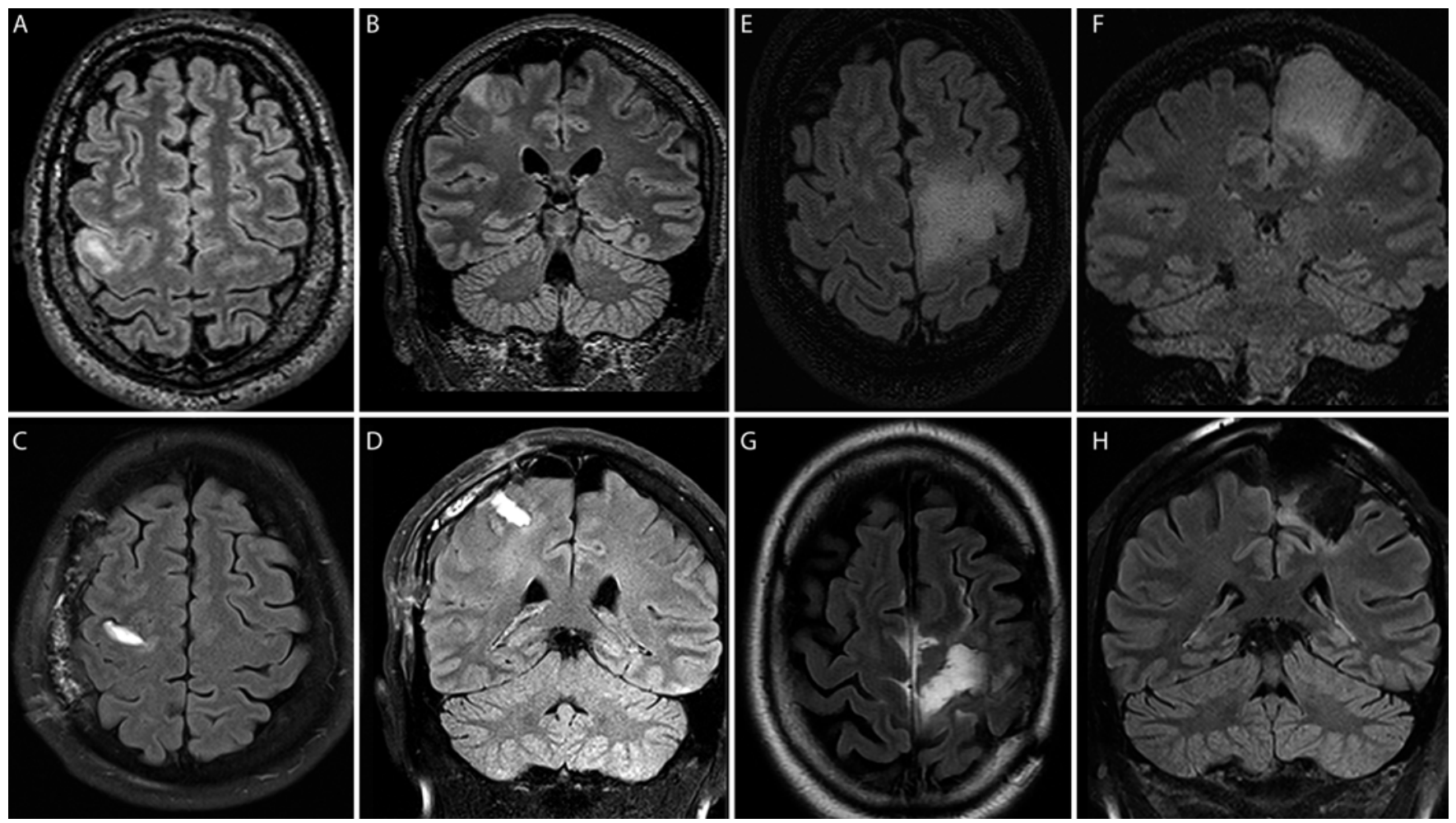

FIG. 5. Example cases of tumor resection. A-D: Preoperative ( $A$ and $B)$ and postoperative $(C$ and $D)$ axial and coronal FLAIR images obtained in a patient with an LGG (IDH1 wildtype, astrocytoma grade II, 1p/19q not deleted) located in the right zone 2 in whom a total resection of the tumor was performed using the reduced train approach (resection cavity shown in $C$ and $D$ ). E-H: Preoperative (E and F) and postoperative ( $G$ and $H$ ) axial and coronal FLAIR images performed in a patient with an LGG (IDH1 mutated, astrocytoma grade II, 1p/19q not deleted) in the left zone 1 in whom a complete resection was obtained using the reduced train approach (resection cavity shown in $\mathrm{G}$ and $\mathrm{H}$ ).

Second, in patients who received previous treatment and harbored either HGGs or metastases, a modification of the standard HF paradigm was required to obtain a reliable cortical and subcortical mapping. In this setting, the excitability of the corticospinal neurons and of the corresponding efferent fibers might have been reduced by the previous treatment. Consequently, to evoke motor responses, a higher charge was required, which was obtained by increasing the number of pulses of the short train, associated in some cases also with an increase in the pulse duration. We preferred to follow this strategy along with inverting the polarity of the stimulation when needed, instead of increasing the current intensity, to reduce the risk of inducing stimulation-related intraoperative seizures. ${ }^{3}$ At the end of the resection, when the tumor mass compression was removed, the excitability of M1 improved, as shown by the fact that the MEPs recorded from the cortical strip were elicited again by the standard To5 paradigm. Despite this, a transient paresis was observed in all patients, as usually occurs when functional boundaries are reached during resection.

Third, in cases of patients with a long clinical history of poorly controlled seizures, harboring tumors with irregular borders, without contrast enhancement (lower grade), possibly not reaching the cortical surface, the standard HF-To5 approach had to be adapted using a reduced-pulse approach. In this clinical condition, the standard To5 para- digm evoked responses from several corticospinal neurons, preventing the disclosure of a clearly defined cortical map for the entry zone at a high resolution, particularly when the tumor did not reach the surface. Shifting to a To2 paradigm to investigate the same cortical area identified some sites in which no MEPs were evoked, where we can begin the resection. At the subcortical level, the strategy was to reach the lowest SMT with both paradigms. While the standard To5 paradigm at $2 \mathrm{~mA}$ elicited MEPs from several muscles, the shift (in the same site) to the To2 paradigm detected MEPs from no or fewer muscles, usually at higher MT (8-10 mA). This allowed us to continue with the resection until an sMT of $2 \mathrm{~mA}$ was reached with this paradigm. This explains the benefit of using the To2 instead of the To5 for establishing the margin of resection, i.e., to continue and extend the resection beyond the site identified with the standard To5 paradigm. The efficacy of this approach is also confirmed by the very low rate of permanent morbidity. However, the underlying neurophysiological mechanism remains unclear. Recent investigations on the functional organization of human M1 have shown some evidence within the primary motor cortex of two separate functional sectors, one anterior and one posterior, characterized by different functional properties and anatomy. ${ }^{13,23}$ The posterior region has been shown to have higher excitability and faster motor output (i.e., motor responses with shorter latency). By modifying the number 
of pulses, we speculate that it may be possible to target corticofugal fibers with different functional properties, allowing for an increase in spatial resolution when tracking the margin of resection, extending tumor removal. The spatial resolution of the stimulation may be increased by switching from a monopolar to a bipolar probe, which has higher focality. This advanced mapping approach requires the continuous assessment of motor pathway integrity, by performing continuous MEP monitoring.

Overall, these data indicate high versatility of the HF stimulation, which allows for reliable mapping in most clinical conditions. Cortically, the assessment of the threshold current is crucial in defining a reliable cortical mapping and identifying a safe cortical entry zone; subcortically, it is crucial to estimate the minimal distance between the functional motor fibers and the probe, which has to be adjusted according to clinical conditions. In this regard, the use of general anesthesia is preferred, because it provides stable cortical excitability, crucial for the evaluation of quantitative parameters (i.e., MEP amplitude-current intensity), which can be quite variable in awake conditions. Shorttrain stimulation is also less ictogenic, even in cases with altered cortical excitability; in addition, the intraoperative use of ECoG for detecting early cortical abnormalities further decreases the risk of inducing intraoperative seizures.

\section{Limitations}

This is a retrospective study and is limited by selection bias. The functional impact was evaluated using the MRC scale. Neuropsychological evaluation was performed as well, although not reported in the present study. The use of HF paradigms proposed in this paper requires some technical nuances, a sophisticated intraoperative machine, careful response interpretation by an in-house experienced neurophysiologist, and a neurophysiology-trained surgeon.

\section{Conclusions}

This study provides evidence that resection of tumors within M1 is feasible and safe when brain mapping is used. The reported experience was performed with HF stimulation, which is highly versatile. The key message is that the stimulation paradigm and probe must be appropriately adapted to clinical context, which then provides the best resection and functional results.

\section{Acknowledgments}

This work was supported by grants ELOQUENTSTIM (no. POR FESR 2014-2020, Regione Lombardia) to Drs. Cerri and Bello, and AIRC (no. 18482) to Dr. Bello.

\section{References}

1. Aboian MS, Solomon DA, Felton E, Mabray MC, VillanuevaMeyer JE, Mueller S, et al: Imaging characteristics of pediatric diffuse midline gliomas with histone H3 K27M mutation. AJNR Am J Neuroradiol 38:795-800, 2017

2. Bello L, Gambini A, Castellano A, Carrabba G, Acerbi F, Fava E, et al: Motor and language DTI fiber tracking combined with intraoperative subcortical mapping for surgical removal of gliomas. Neuroimage 39:369-382, 2008

3. Bello L, Riva M, Fava E, Ferpozzi V, Castellano A, Raneri
F, et al: Tailoring neurophysiological strategies with clinical context enhances resection and safety and expands indications in gliomas involving motor pathways. Neuro Oncol 16:1110-1128, 2014

4. Buckner J, Giannini C, Eckel-Passow J, Lachance D, Parney I, Laack N, et al: Management of diffuse low-grade gliomas in adults-use of molecular diagnostics. Nat Rev Neurol 13:340-351, 2017

5. De Witt Hamer PC, Robles SG, Zwinderman AH, Duffau $\mathrm{H}$, Berger MS: Impact of intraoperative stimulation brain mapping on glioma surgery outcome: a meta-analysis. J Clin Oncol 30:2559-2565, 2012

6. Duffau H, Mandonnet E: The "onco-functional balance" in surgery for diffuse low-grade glioma: integrating the extent of resection with quality of life. Acta Neurochir (Wien) 155:951-957, 2013

7. Han SJ, Morshed RA, Troncon I, Jordan KM, Henry RG, Hervey-Jumper SL, et al: Subcortical stimulation mapping of descending motor pathways for perirolandic gliomas: assessment of morbidity and functional outcome in 702 cases. J Neurosurg 131:201-208, 2019

8. Ius T, Angelini E, Thiebaut de Schotten M, Mandonnet E, Duffau $\mathrm{H}$ : Evidence for potentials and limitations of brain plasticity using an atlas of functional resectability of WHO grade II gliomas: towards a "minimal common brain." Neuroimage 56:992-1000, 2011

9. Magill ST, Han SJ, Li J, Berger MS: Resection of primary motor cortex tumors: feasibility and surgical outcomes. J Neurosurg 129:961-972, 2018

10. Noell S, Feigl GC, Naros G, Barking S, Tatagiba M, Ritz $\mathrm{R}$ : Experiences in surgery of primary malignant brain tumours in the primary sensori-motor cortex practical recommendations and results of a single institution. Clin Neurol Neurosurg 136:41-50, 2015

11. Obermueller T, Schaeffner M, Gerhardt J, Meyer B, Ringel F, Krieg SM: Risks of postoperative paresis in motor eloquently and non-eloquently located brain metastases. BMC Cancer 14:21, 2014

12. Penfield W, Boldrey E: Somatic motor and sensory representation in the cerebral cortex of man as studied by electrical stimulation. Brain 60:389-443, 1937

13. Rathelot JA, Strick PL: Subdivisions of primary motor cortex based on cortico-motoneuronal cells. Proc Natl Acad Sci U S A 106:918-923, 2009

14. Rossi M, Fornia L, Puglisi G, Leonetti A, Zuccon G, Fava E, et al: Assessment of the praxis circuit in glioma surgery to reduce the incidence of postoperative and long-term apraxia: a new intraoperative test. J Neurosurg 130:17-27, 2018

15. Rossi M, Sani S, Nibali MC, Fornia L, Bello L, Byrne RW: Mapping in low-grade glioma surgery: low- and highfrequency stimulation. Neurosurg Clin N Am 30:55-63, 2019

16. Sanai N, Berger MS: Glioma extent of resection and its impact on patient outcome. Neurosurgery 62:753-764, 264-266, 2008

17. Schucht P, Seidel K, Beck J, Murek M, Jilch A, Wiest R, et al: Intraoperative monopolar mapping during 5-ALA-guided resections of glioblastomas adjacent to motor eloquent areas: evaluation of resection rates and neurological outcome. Neurosurg Focus 37(6):E16, 2014

18. Seidel K, Beck J, Stieglitz L, Schucht P, Raabe A: Lowthreshold monopolar motor mapping for resection of primary motor cortex tumors. Neurosurgery 71 (1 Suppl Operative): $104-115,2012$

19. Smith JS, Chang EF, Lamborn KR, Chang SM, Prados MD, Cha S, et al: Role of extent of resection in the long-term outcome of low-grade hemispheric gliomas. J Clin Oncol 26:1338-1345, 2008

20. Szelényi A, Bello L, Duffau H, Fava E, Feigl GC, Galanda 
M, et al: Intraoperative electrical stimulation in awake craniotomy: methodological aspects of current practice. Neurosurg Focus 28(2):E7, 2010

21. Szelényi A, Senft C, Jardan M, Forster MT, Franz K, Seifert V, et al: Intra-operative subcortical electrical stimulation: a comparison of two methods. Clin Neurophysiol 122:14701475,2011

22. van den Bent MJ, Wefel JS, Schiff D, Taphoorn MJB, Jaeckle $\mathrm{K}$, Junck L, et al: Response assessment in neuro-oncology (a report of the RANO group): assessment of outcome in trials of diffuse low-grade gliomas. Lancet Oncol 12:583-593, 2011

23. Viganò L, Fornia L, Rossi M, Howells H, Leonetti A, Puglisi $\mathrm{G}$, et al: Anatomo-functional characterisation of the human "hand-knob": a direct electrophysiological study. Cortex 113:239-254, 2019

\section{Disclosures}

The authors report no conflict of interest concerning the materials or methods used in this study or the findings specified in this paper.

\section{Author Contributions}

Conception and design: Bello. Acquisition of data: Rossi, Conti Nibali. Analysis and interpretation of data: Rossi, Viganò, Bello. Drafting the article: Conti Nibali, Howells, Sciortino, Bello. Critically revising the article: Gay, Leonetti, Riva, Fornia. Reviewed submitted version of manuscript: Howells. Statistical analysis: Puglisi. Study supervision: Cerri, Bello.

\section{Correspondence}

Marco Rossi: Humanitas Research Hospital, Milan, Italy. rossi. marco@gmail.com. 